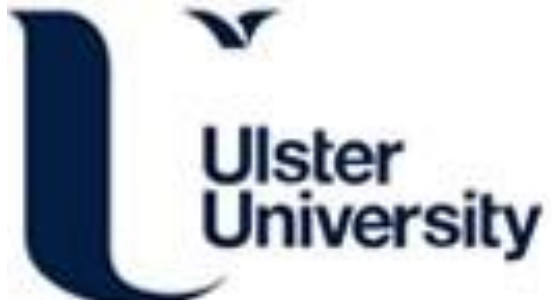

\section{Kinematic Differences in Shoulder Roll and Hip Roll at Different Front Crawl Speeds in National Level Swimmers}

Andersen , J., Sinclair, P., McCabe, C., \& Sanders, R. (2020). Kinematic Differences in Shoulder Roll and Hip Roll at Different Front Crawl Speeds in National Level Swimmers. Journal of Strength and Conditioning Research, 34(1), 20-25. https://doi.org/10.1519/JSC.0000000000003281

Link to publication record in Ulster University Research Portal

Published in:

Journal of Strength and Conditioning Research

Publication Status:

Published (in print/issue): 01/01/2020

DOI:

10.1519/JSC.0000000000003281

\section{Document Version}

Author Accepted version

\section{General rights}

Copyright for the publications made accessible via Ulster University's Research Portal is retained by the author(s) and / or other copyright owners and it is a condition of accessing these publications that users recognise and abide by the legal requirements associated with these rights.

\section{Take down policy}

The Research Portal is Ulster University's institutional repository that provides access to Ulster's research outputs. Every effort has been made to ensure that content in the Research Portal does not infringe any person's rights, or applicable UK laws. If you discover content in the Research Portal that you believe breaches copyright or violates any law, please contact pure-support@ulster.ac.uk. 
Differences in shoulder roll and hip roll: Implications for strength training 1

Title: Kinematic differences in shoulder roll and hip roll at different front crawl speeds

Running head: Shoulder and hip roll during front crawl swimming at different speeds

Research conducted at: The University of Edinburgh; The University of Sydney

Authors: Jordan T Andersen ${ }^{1}$, Peter J Sinclair ${ }^{1}$, Carla B McCabe ${ }^{2}$, Ross H Sanders ${ }^{1}$

${ }^{1}$ Physical Activity, Lifestyle, Ageing and Wellness; Faculty of Health Sciences; The University of Sydney

${ }^{2}$ Sport and Exercise Sciences Research Institute; School of Sport; Faculty of Life \& Health Sciences; Ulster University

\section{Corresponding Author:}

Jordan T Andersen

75 East St.

Sydney, NSW, AUSTRALIA

2141

M: +61 410383887

E: jordan.andersen@sydney.edu.au

No funding was received for this work. 
Pages, including References and Figure Legends)

1

2

3

4

5

7

8

Shoulder and hip roll during front crawl swimming at different speeds 1

1 Title: Kinematic differences in shoulder roll and hip roll at different front crawl speeds:

2 Implications for torso muscle demands and dry land strength training in swimming

3

$4 \quad$ Running head: Shoulder and hip roll during front crawl swimming at different speeds 
5 Dry-land strength training is a common component of swimming programs; however, its efficacy

6 is contentious. A common criticism of dry-land strength training for swimming is a lack of

7 specificity. An understanding of movement patterns in swimming can enable dry-land strength

8 training programs to be developed to elicit adaptations that transfer to improvements in swimming

9 performance. This study aimed to quantify the range and velocity of hip roll, shoulder roll, and

10 torso twist (produced by differences in the relative angle between shoulder roll and hip roll) in

11 front crawl at different swimming speeds. Longitudinal torso kinematics were compared between

12 sprint and $400 \mathrm{~m}$ pace front crawl using 3D kinematics of thirteen elite Scottish front crawl 13 specialists. The range (sprint: $78.1^{\circ} ; 400 \mathrm{~m}: 61.3^{\circ}$ ) and velocity of torso twist (sprint: $166.3^{\circ} / \mathrm{s}$;

$14400 \mathrm{~m}: 96.9^{\circ} / \mathrm{s}$ ) were greater at sprint than $400 \mathrm{~m}$ pace. These differences were attributed to 15 reductions in hip roll (sprint: $36.8^{\circ} ; 400 \mathrm{~m}: 49.9^{\circ}$ ) without corresponding reductions in shoulder 16 roll (sprint: $97.7^{\circ} ; 400 \mathrm{~m}: 101.6^{\circ}$ ) when participants swam faster. Shoulder roll velocity (sprint: $17190.9^{\circ} / \mathrm{s} ; 400 \mathrm{~m}: 139.2 \%$ ) and hip roll velocity (sprint: $75.5 \%$ s; 400m: $69.1 \%$ s) were greater at sprint 18 than $400 \mathrm{~m}$ pace due to a higher stroke frequency at sprint pace (sprint: 0.95 strokes $/ \mathrm{s} ; 400 \mathrm{~m}: 0.70$ 19 strokes/s). These findings imply that torques acting to rotate the upper torso and the lower torso 20 are greater at sprint than $400 \mathrm{~m}$ pace. Dry-land strength training specificity can be improved by 21 designing exercises that challenge the torso muscles to reproduce the torques required to generate 22 the longitudinal kinematics in front crawl.

23 Keywords: torso twist, biomechanics, sprint, middle-distance, performance 
Shoulder and hip roll during front crawl swimming at different speeds 3

\section{INTRODUCTION}

25 To maximise the probability that strength training adaptations will transfer to improvements in

26 performance, training must be based on the demands of a sport $(12,13)$. The lack of

27 effectiveness of many dry-land strength training programs in improving swimming performance

28 is often attributed to a lack of specificity in training $(11,32,33)$. Transference of strength

29 training gains to performance can be enhanced by designing exercises that match the demands

30 associated with the movement patterns used within a sport (37). Dry-land strength training

31 specificity for swimming can therefore be improved with a better understanding of the

32 movement patterns used in swimming.

34 Longitudinal body rotation is essential for maximising performance in front crawl swimming (6,

35 17). Rotation of the shoulders and hips about the body's longitudinal axis, known respectively as

36 shoulder roll and hip roll, depend on swimming speed (27). Some characteristics of shoulder roll

37 and hip roll remain consistent across different front crawl speeds; for example, the shoulders roll

38 through a greater range of motion than the hips regardless of swimming speed $(3,35)$. The effect

39 of swimming speed on several features of longitudinal rotation in front crawl, however, remain

40 unclear. For example, it is unknown how torso twist produced by differences in the relative

41 angles of hip roll and shoulder roll varies with swimming speed. Further, the influence of

42 swimming speed on the rate of change (or velocity) of hip roll, shoulder roll, and torso twist has

43 never been reported. Considering the association between torso muscle activity and the

44 magnitude and speed of twisting motions of the spine $(16,19)$, differences in the range and

45 velocity of torso twist in front crawl may influence the demands on the torso muscles. Our 
46 understanding of the torso muscle requirements in front crawl may therefore be limited by the

47 lack of evidence of torso twist characteristics in front crawl swimming.

49 Total hip roll, a measurement of the range of hip roll from one side to the other, tends to decrease

50 as swimming speed increases while total shoulder roll, which is the range of shoulder roll from

51 one side to the other, does not seem to change with increasing speed as much as total hip roll.

52 McCabe and Sanders (21) reported a total hip roll of 57 degrees at $1.50 \mathrm{~m} / \mathrm{s} \mathrm{during}$ a $400 \mathrm{~m}$

53 maximal effort while Psycharakis and Sanders (26) reported a total hip roll of 44 degrees at 1.68

$54 \mathrm{~m} / \mathrm{s}$ in the first $50 \mathrm{~m}$ of a $200 \mathrm{~m}$ maximal front crawl test. Psycharakis and McCabe (25) reported

55 an even lower total hip roll of 39 degrees at $1.81 \mathrm{~m} / \mathrm{s}$ during a maximal $25 \mathrm{~m}$ sprint. Despite the

5618 degrees difference in total hip roll between 400m pace and sprint front crawl swimming, total

57 shoulder roll remained between 105 and 111 degrees across all three studies. Differences in the

58 range and/or timing of hip roll and shoulder roll require twist within the torso. Data from the

59 studies by McCabe and Sanders (21), Psycharakis and Sanders (26), and Psycharakis and

60 McCabe (25) indicate that the range of torso twist is likely to increase with swimming speed;

61 however, differences in torso twist from the same group of swimmers swimming at different

62 front crawl speeds have never been examined.

63

64 The time for the hips and shoulders to roll from one side to the other and back again is

65 determined by the duration of the arm stroke cycle $(28,34)$. The velocities of hip roll and

66 shoulder roll are therefore influenced by the range of hip roll and shoulder roll, respectively, and

67 the number of stroke cycles per unit of time, or stroke frequency. It is well documented that 
68 stroke frequency increases as swimming speed increases $(4,7,29,30)$; however, the influence of

69 swimming speed on hip roll velocity and shoulder roll velocity is unknown. Changes in the

70 relative angle between hip roll and shoulder roll and differences in stroke frequency across front

71 crawl speeds suggest that torso twist velocity may also change with swimming speed, but torso

72 twist velocity has yet to be quantified in the scientific literature.

73

74 Although twist of the shoulders and hips relative to each other is influenced by the torques

75 produced by the actions of the upper and lower limbs, it may be hypothesised that the differences

76 between shoulder and hip rotation, manifest in changing torso twist angles, is also influenced by

77 the actions of the torso muscles connecting the shoulders and hips. Therefore, it is likely that

78 differences in torso twist rates of change, that is, torso twist velocities, may reflect differences in

79 demands on the torso muscles to control posture and maintain stability of the swimmer's torso.

80 Further, if there are differences in the relative magnitudes and velocities of shoulder and hip roll

81 between paces, demands on the torso muscles are likely to differ between swimming speeds.

82 Therefore, insights into these demands may be gained by quantifying the differences in the range 83 and velocity of torso twist at different swimming paces.

85 While the ranges of hip roll and shoulder roll at different swimming speeds have been examined

86 in separate studies, the differences in the velocities of hip roll and shoulder roll between

87 swimming speeds have never been reported. Moreover, the range and velocity of torso twist

88 produced by differences in hip and shoulder roll at different front crawl speeds have never been

89 examined to our knowledge. These gaps in swimming research present a barrier to understanding 
90 the movement patterns in front crawl swimming that can be used to improve the specificity of

91 dry-land strength training for swimmers. Therefore, the purpose of this study was to quantify the

92 range and velocity of hip roll, shoulder roll, and torso twist in front crawl at different swimming

93 speeds. The differences in the longitudinal kinematics between speeds will further our

94 understanding of the movement patterns in front crawl swimming which can be used to develop

95 insight into the demands on the torso muscles in front crawl swimming.

97 METHODS

98 Experimental Approach to the Problem

99 This cross-sectional study of three-dimensional kinematics enabled analysis of the movement 100 patterns of high level front crawl swimming for two different event distances (i.e. $50 \mathrm{~m}$ and $400 \mathrm{~m}$ 101 freestyle). National and international level swimmers were recruited because of their ability to 102 produce movement patterns that can provide insights into the requirements for high level 103 swimming performance. While experienced swimmers are known to reliably produce consistent 104 swimming technique, multiple trials at both swimming paces were collected to account for 105 individual variability inherent of human movement.

108 Three-dimensional coordinate data of a 15 segment whole-body model of thirteen national and 109 international level male Scottish front crawl specialists (age: $17.54 \pm 1.98$ years, range 15 to 22 years; height: $181.18 \pm 4.98 \mathrm{~cm}$; weight: $71.58 \pm 6.26 \mathrm{~kg}$ ) were analysed from a data set that was 
111 previously utilized in the studies of McCabe, Psycharakis and Sanders (20) and McCabe and 112 Sanders (21). Participants had specialized in front crawl for a minimum of two years, were not 113 currently injured or recovering from injury, and held a short course personal best time of either 114 less than 24.60 s for $50 \mathrm{~m}$ or less than $4 \mathrm{~min} 10$ s for $400 \mathrm{~m}$. The protocols and procedures were 115 approved by the university ethics committee. All participants were informed of the risks and 116 benefits of the study and provided written consent prior to data collection. For participants under 117 the age of 18, participants and a parent or guardian provided written consent.

119 Procedures

120 The data collection by McCabe was conducted in an indoor 25m pool. Participants were marked 121 to enable identification of the following anatomical landmarks: the vertex of the head (on top of 122 the swim cap), the left and right: tip of the 3rd distal phalanx of the finger, wrist axis, elbow axis, 123 shoulder axis, hip axis, knee axis, ankle axis, lateral aspect of the 5th metatarsophalangeal joint, 124 and tip of 1st phalanx of the foot (big toe). After an individualized warm up, participants swam $1254 \times 25 \mathrm{~m}$ at sprint pace and one $400 \mathrm{~m}$ effort at a pace that would result in the fastest time possible. 126 After each sprint trial, participants swam back to the start position at recovery pace and rested in127 water for two minutes before beginning the next trial. The order of swimming pace was 128 randomized and participants swam for at least five minutes to recover after completing the first 129 pace, then exited the pool for an additional ten minute rest before warming up again and 130 completing the second pace. 
132 As participants swam through a calibration volume (4.5m long, $1.0 \mathrm{~m}$ wide, and 1.5 in height)

133 located $15.25 \mathrm{~m}$ from the starting wall, their motion was captured by six synchronized JVC KY32

$134 \mathrm{CCD}$ cameras (four below and two above the water surface) at a frame rate of $50 \mathrm{~Hz}$. Each trial

135 began from a push start and participants were required to not breathe as they swam through the

136 calibration volume to avoid any effect of the breathing actions on their swimming technique (25,

137 30). Swimmers familiarized themselves with the breath-holding requirement during warm up.

138 All participants used a six-beat flutter kick at both swimming paces.

140 Data Processing

141 One stroke cycle (SC) was defined as the moment the tip of the third digit of one hand entered 142 the water to the subsequent entry of that digit on the same hand performed completely within the 143 calibrated space. At sprint pace, one SC was analysed for each of the four $25 \mathrm{~m}$ trials. During the $144400 \mathrm{~m}$ effort, one SC was recorded from the first $25 \mathrm{~m}$ length of each 50m lap. SCs from laps 2, 3, 1454 and 5 during the $400 \mathrm{~m}$ effort were analysed, totaling four observations per swimmer at $400 \mathrm{~m}$ 146 pace. These laps were selected to align with previous findings that laps 1,7 , and 8 were 147 consistently different from laps 2-6 (21). Lap 6 was excluded to further minimize the effect of 148 fatigue on swimming technique. Due to marker occlusion during data collection that prevented 149 digitization of landmarks over several consecutive frames, one trial from one participant at 400m 150 pace (P4) was discarded. Data were retained for all four trials at both paces from every other 151 participant. 
153 Three-dimensional reconstruction from manual digitization of the anatomical landmarks was 154 conducted using the Ariel Performance Analysis System (direct linear transformation algorithms 155 from Abdel-Aziz and Karara (1)). Errors due to digitization for the variables used in the current 156 study were considered small from digitization reliability tested in a previous study (20). To 157 prevent data loss during filtering, an additional 30 frames were extrapolated by reflection. 158 Fourier truncation was used to filter the position data of the body landmarks. This filtering 159 strategy was deemed appropriate because the cyclic nature of movements in front crawl 160 swimming results in periodic data (2). Residual analysis indicated that a $6 \mathrm{~Hz}$ cut-off was 161 suitable to smooth the data. SC length was then standardized to 201 points using a Fourier 162 transform and inverse transform so that each datum represented a half percentage of the SC (i.e. $1630-100 \%)$.

165 The filtered anatomical landmark data were entered into a bespoke MATLAB (Mathworks, Inc.) 166 analysis program written by the last author. The orthogonal external reference system was 167 defined by the horizontal $\mathrm{X}$-axis pointing in the swimming direction, the $\mathrm{Y}$-axis pointing 168 vertically up, and the horizontal Z-axis pointing to the swimmer's right. Shoulder roll and hip 169 roll were calculated independently for each percentile of the SC as the angle, expressed in 170 degrees, between the Z-axis and vectors connecting the shoulders and hips, respectively, 171 projected onto the $\mathrm{YZ}$ plane. 
178 Stroke frequency was determined using the inverse of the time to complete one SC (stroke/s).

179 Torso twist was the difference in the relative angles of shoulder roll and hip roll and was

180 calculated for each percentile of the SC in degrees. Hip roll velocity, shoulder roll velocity, and

181 torso twist velocity were the rate of change of hip roll, shoulder roll, and torso twist,

182 respectively, and were expressed as angular velocities (in degrees per second) using the time

183 derivatives of hip roll, shoulder roll, and torso twist with the central difference method.

185 Range of hip roll, range of shoulder roll, and range of torso twist were determined separately for 186 each trial by summing the maximum magnitude of hip roll, shoulder roll, and torso twist, 187 respectively, to the left side and to the right side. Averages for hip roll velocity, shoulder roll 188 velocity, and torso twist velocity were calculated using the mean of the absolute values of hip 189 roll velocity, shoulder roll velocity, and torso twist velocity, respectively, over each entire SC.

191 Statistical Analyses

192 Statistical tests were performed using IBM SPSS Statistics $24(\alpha=0.05)$, with the exception of 193 effect sizes which were calculated manually (10). Intra-class correlations between swimming 194 trials were determined using a single-rating, absolute agreement, two-way mixed random effects 
model analysis (14) for stroke frequency, range of hip roll, range of shoulder roll, range of torso twist, average hip roll velocity, average shoulder roll velocity, and average torso twist velocity at sprint pace and $400 \mathrm{~m}$ pace.

Means and $95 \%$ confidence intervals (i.e. the $t$-value for the sample size $(n=13)$ multiplied by 211 software $\left(\mathrm{G}^{*}\right.$ Power 3.1) (9). the standard error of the sample mean) were calculated at both swimming paces for stroke frequency, range of hip roll, range of shoulder roll, range of torso twist, average hip roll velocity, average shoulder roll velocity, and average torso twist velocity. Confidence intervals improved our ability to compare and interpret differences between swimming paces by providing a range about the mean of each kinematic variable in which the true mean was likely to fall for either pace. The Shapiro-Wilk test indicated that all variables were normally distributed. Separate paired $t$-test were conducted to evaluate the differences in stroke frequency, range of hip roll, range of shoulder roll, range of torso twist, average hip roll velocity, average shoulder roll velocity, and average torso twist velocity between sprint pace and $400 \mathrm{~m}$ pace. Effect sizes were determined using Cohen's $d$ and interpreted with the following recommendations: small 0.2, moderate 0.5 , and large 0.8 (5). Post hoc power analysis was conducted using open-source

\section{RESULTS}

Intra-class correlations were high for stroke frequency (sprint pace: $0.93 ; 400 \mathrm{~m}$ pace: 0.98 ) range of hip roll (sprint pace: $0.90 ; 400 \mathrm{~m}$ pace: 0.93 ), range of shoulder roll (sprint pace: $0.85 ; 400 \mathrm{~m}$ pace: 0.94$)$, range of torso twist (sprint pace: $0.82 ; 400 \mathrm{~m}$ pace: 0.91 ), average hip roll velocity 
7

8

(sprint pace: $0.90 ; 400 \mathrm{~m}$ pace: 0.91 ), average shoulder roll velocity (sprint pace: $0.83 ; 400 \mathrm{~m}$

pace: 0.96 ), and average torso twist velocity (sprint pace: $0.84 ; 400 \mathrm{~m}$ pace: 0.89 ) at both paces.

220 Time series for ensemble averages of hip roll, shoulder roll, and torso twist are shown in Figure 2211 and time series for ensemble averages of hip roll velocity, shoulder roll velocity, torso twist 222 velocity are shown in Figure 2 for one SC at sprint and 400m pace.

224 [INSERT FIGURE 1 NEAR HERE]

225 [INSERT FIGURE 2 NEAR HERE]

227 Table 1 shows means, 95\% confidence intervals using the $t$-distribution of the sample mean, 228 effect sizes, and statistical power for comparisons between paces of stroke frequency, range of 229 hip roll, range of shoulder roll, range of torso twist, average hip roll velocity, average shoulder 230 roll velocity, and average torso twist velocity. Stroke frequency was greater at sprint pace than at $231400 \mathrm{~m}$ pace $(t(12)=12.27, p<0.01)$ with a large effect size. Range of hip roll was greater at $232400 \mathrm{~m}$ pace than at sprint pace $(t(12)=6.77, p<0.01)$ with a large effect size while range of 233 shoulder roll was similar between paces $(p=0.14)$. Range of torso twist $(t(12)=6.88, p<0.01)$, 234 average shoulder roll velocity $(t(12)=9.17, p<0.01)$, and average torso twist velocity $(t(12)=$ $23512.30, p<0.01)$ were greater at sprint pace than at $400 \mathrm{~m}$ pace with large effect sizes. Average 236 hip roll velocity was also greater at sprint pace than at $400 \mathrm{~m}$ pace $(t(12)=2.98, p<0.05)$ but 237 with a moderate effect size. 


\section{[INSTERT TABLE 1 NEAR HERE]}

\section{DISCUSSION}

The purpose of this study was to quantify the range and velocity of hip roll, shoulder roll, and torso twist in front crawl at different swimming speeds. The differences in hip roll and shoulder roll that contributed to the changes in the range and velocity of torso twist between paces will further understanding of the demands on the torso muscles in front crawl swimming. The findings from this study contribute to the knowledge of movement patterns in front crawl that can be used to improve the specificity of dry-land strength training for swimmers.

\section{The larger range of torso twist at sprint pace than at $400 \mathrm{~m}$ pace seemed to be the result of a} reduction in hip roll without a corresponding reduction in shoulder roll when participants were swimming faster. The range of hip roll and range of shoulder roll observed in the current study are consistent with trends of total hip roll and total shoulder roll across different swimming speeds from previous findings (26). The similar range of shoulder roll between paces and the higher stroke frequency at sprint pace than at 400m pace meant the swimmers rolled their shoulders faster as swimming speed increased. This was reflected in an average shoulder roll velocity that was $37 \%$ greater at sprint pace than at $400 \mathrm{~m}$ pace (Table 1). Despite the smaller range of hip roll at sprint pace than at $400 \mathrm{~m}$ pace, the higher stroke frequency resulted in an increase in hip roll velocity as swimming speed increased; however, average hip roll velocity 
259 was only $9 \%$ greater at sprint pace than at $400 \mathrm{~m}$ pace. Moreover, the effect size of the difference

260 in average hip roll velocity was moderate while all other statistically significant differences

261 between paces had large effect sizes (Table 1). The difference in torso twist velocity between

262 swimming paces therefore seemed to be the result of the swimmers' ability to maintain their 263 range of shoulder roll, despite an increase in stroke frequency, and to reduce their range of hip 264 roll as they increased swimming speed.

266 The patterns of hip roll, shoulder roll, and torso twist in Figure 1 suggest the magnitude of 267 rotation between the upper and lower torso was greater at sprint pace than at $400 \mathrm{~m}$ pace.

268 Furthermore, the difference in torso twist velocity between swimming paces implies the 269 swimmers in this study rotated their upper torso with respect to their lower torso more rapidly at 270 sprint pace than at $400 \mathrm{~m}$ pace. Increases in the magnitude and speed of rotation between the 271 upper and lower torso are associated with higher torso muscle activity $(18,19)$. These findings 272 suggest that the demands on the torso muscles are likely to be higher at faster swimming speeds 273 but this cannot be stated with confidence without further research measuring the muscle activity 274 at different paces.

276 Torques that produce rotation of the upper torso must have been higher at sprint pace than at $277400 \mathrm{~m}$ pace in order for the swimmers to achieve a similar range of shoulder roll at both paces 278 considering the increase in stroke frequency as swimming speed increased. Hydrodynamic and 279 buoyancy torques associated with the arm stroke produce longitudinal body rotation $(23,34,36)$ 280 and could have contributed to the differences in shoulder roll velocity observed in the current 
281 study. Though the shoulders and hips roll somewhat independently in front crawl (28),

282 longitudinal rotation is likely transferred from the shoulders to the hips. For example, motion can

283 be transferred along the torso during twisting motions of the spine through passive mechanisms

284 (e.g. via connective tissue and intervertebral discs) $(15,16)$ or with the assistance of muscle

285 torques $(19,24,31)$. Greater torque acting to rotate the lower torso, separate from the torques

286 acting to rotate the upper torso, may have therefore been required to reduce the range of hip roll

287 as swimming speed increased. Sanders and Psycharakis (28), for instance, hypothesized that hip

288 roll is "dampened" compared to shoulder roll from torques associated with the flutter kick.

289 Considering swimmers tend to increase kicking frequency as swimming speed increases $(4,8$,

290 22), torques from the flutter kick acting on the lower torso may have been greater at sprint pace

291 than at $400 \mathrm{~m}$ pace, which could have contributed to the reduction in hip roll as swimming speed

292 increased. The differences in the longitudinal kinematics presented here indicate that the torques

293 acting to rotate the upper torso and the torques acting to rotate the lower torso may be greater at

294 sprint pace than at $400 \mathrm{~m}$ pace. This may also indicate that the demands on the torso muscles

295 increase as swimming speed increases. Quantification of the torques acting on the upper torso

296 and lower torso in front crawl is required to test this hypothesis.

\section{PRACTICAL APPLICATIONS}

299 This is the first study to investigate the velocity of hip roll, shoulder roll, and torso twist in front

300 crawl swimming. Coaches can use these findings to guide recommendations for changes to

301 swimming technique between sprint and middle-distance swimming. For example, swimmers

302 can be encouraged to maintain their range of shoulder roll as stroke frequency increases with 
303 swimming speed. From the differences in the range and velocity of torso twist between

304 swimming paces, torques acting to produce rotation of the upper torso and the lower torso are

305 likely to increase as swimming speed increases. Dry-land strength training specificity may be

306 improved by designing exercises that challenge the torso muscles to generate torques that

307 produce or resist longitudinal rotation of the upper torso and the lower torso. Coaches are

308 encouraged to consider the differences in the demands placed on swimmers competing over

309 different distances when designing dry-land strength training. For instance, torques required

310 from the torso muscles may be greater at faster swimming speeds than at slower swimming

311 speeds. As swimming speed increases, exercises should increase the amount of torque required

312 from the torse muscle. This Acknowledgement of the differences in demands between swimming

313 speeds could increase the likelihood that benefits from dry-land strength training will transfer to

314 improvements in swimming performance.

316 ACKNOWLEDGMENTS

317 The authors would like to thank the swimmers and coaches for their participation in data

318 collection. There are no conflicts of interest to declare.

320 REFERENCES

321 1. Abdel-Aziz Y and Karara H. Direct linear transformation from comparator coordinates 322 into object space coordinates in close-range photogrammetry. Presented at Proceedings of 323 the symposium on close-range photogrammetry, Falls Church, VA, 1971.

3242 2. Bartlett R. Introduction to sports biomechanics: Analysing human movement patterns. $325 \quad$ Routledge, 2007. 
3. Cappaert JM, Pease DL, and Troup JP. Three-Dimensional Analysis of the Men's 100-m Freestyle during the 1992 Olympic Games. J Appl Biomech 11: 103-112, 1995.

4. Chollet D, Chalies S, and Chatard JC. A new index of coordination for the crawl: description and usefulness. Int J Sports Med 21: 54-59, 2000.

5. Cohen J. Statistical power analysis for the behavioral sciences 2nd edn. Erlbaum Associates, Hillsdale, 1988.

6. Counsilman JE and Counsilman BE. The new science of swimming. Englewood Cliffs, NJ: Prentice-Hall Inc., 1994.

7. Craig AB, Skehan PL, Pawelczyk JA, and Boomer WL. Velocity, stroke rate, and distance per stroke during elite swimming competition. Med Sci Sports Exerc 17: 625634, 1985.

8. de Jesus K, Sanders RH, de Jesus K, Ribeiro J, Figueiredo P, Vilas-Boas JP, and Fernandes RJ. The effect of intensity on 3-dimensional kinematics and coordination in front-crawl swimming. International Journal of Sports Physiology and Performance 11: 768-775, 2016.

9. Faul F, Erdfelder E, Lang A-G, and Buchner A. G*Power 3: A flexible statistical power analysis program for the social, behavioral, and biomedical sciences. Behavior Research Methods 39: 175-191, 2007.

10. Fritz CO, Morris PE, and Richler JJ. Effect size estimates: current use, calculations, and interpretation. Journal of Experimental Psychology: General 141: 2, 2012.

11. Girold S, Maurin D, Dugue B, Chatard J-C, and Millet G. Effects of dry-land vs. resistedand assisted-sprint exercises on swimming sprint performances. Journal of Strength and Conditioning Research 21: 599, 2007.

12. González-Badillo JJ and Sánchez-Medina L. Movement velocity as a measure of loading intensity in resistance training. Int J Sports Med 31: 347-352, 2010.

13. Izquierdo M, Häkkinen K, Gonzalez-Badillo JJ, Ibanez J, and Gorostiaga EM. Effects of long-term training specificity on maximal strength and power of the upper and lower extremities in athletes from different sports. European journal of applied physiology 87: 264-271, 2002.

14. Koo $\mathrm{T}$ and $\mathrm{Li} \mathrm{M}$. A guideline of selecting and reporting intraclass correlation coefficients for reliability research. J Chiropr Med. 2016; 15 (2): 155-63. Epub 2016/03/31. 10.1016/j. jcm. 2016.02. 012. https://doi. org/10.1016/j. jcm. 2016.02. 012 PMID: $27330520,2017$.

15. Kumar S, Narayan Y, and Garand D. An electromyographic study of isokinetic axial rotation in young adults. The Spine Journal 3: 46-54, 2003.

16. Kumar S, Narayan Y, and Zedka M. An electromyographic study of unresisted trunk rotation with normal velocity among healthy subjects. Spine 21: 1500-1512, 1996.

17. Maglischo EW. Swimming fastest. Champaign, Ill.: Human Kinetics, 2003.

18. Marras W, Davis K, and Granata K. Trunk muscle activities during asymmetric twisting motions. Journal of Electromyography and Kinesiology 8: 247-256, 1998.

19. Marras WS and Granata KP. A biomechanical assessment and model of axial twisting in the thoracolumbar spine. Spine 20: 1440-1451, 1995.

20. McCabe CB, Psycharakis S, and Sanders RH. Kinematic differences between front crawl sprint and distance swimmers at sprint pace. Journal of Sports Sciences 29: 115-123, 2011. 
371 21. McCabe CB and Sanders RH. Kinematic differences between front crawl sprint and distance swimmers at a distance pace. Journal of Sports Sciences 30: 601-608, 2012.

22. Millet G, Chollet D, Chalies S, and Chatard J. Coordination in front crawl in elite triathletes and elite swimmers. Int J Sports Med 23: 99-104, 2002.

23. Payton CJ, Bartlett RM, Baltzopoulos V, and Coombs R. Upper extremity kinematics and body roll during preferred-side breathing and breath-holding front crawl swimming. Journal of sports sciences 17: 689-696, 1999.

24. Pink M, Perry J, and Jobe FW. Electromyographic analysis of the trunk in golfers. The american journal of sports medicine 21: 385-388, 1993.

25. Psycharakis $\mathrm{S}$ and McCabe C. Shoulder and hip roll differences between breathing and non-breathing conditions in front crawl swimming. Journal of Biomechanics 44: 17521756, 2011.

26. Psycharakis S and Sanders RH. Shoulder and hip roll changes during 200-m front crawl swimming. Medicine \& Science in Sports \& Exercise 40: 2129-2136, 2008.

27. Psycharakis $\mathrm{S}$ and Sanders RH. Body roll in swimming: A review. Journal of Sports Sciences 28: 229-236, 2010.

28. Sanders RH and Psycharakis SG. Rolling rhythms in front crawl swimming with six-beat kick. Journal of Biomechanics 42: 273-279, 2009.

29. Schnitzler C, Seifert L, Ernwein V, and Chollet D. Arm coordination adaptations assessment in swimming. Int J Sports Med 29: 480-486, 2008.

30. Seifert L, Chollet D, and Bardy B. Effect of swimming velocity on arm coordination in the front crawl: a dynamic analysis. Journal of sports sciences 22: 651-660, 2004.

31. Shaffer B, Jobe FW, Pink M, and Perry J. Baseball Batting: An Electromyographic Study. Clinical orthopaedics and related research 292: 285-293, 1993.

32. Tanaka H, Costill DL, Thomas R, Fink WJ, and Widrick JJ. Dry-land resistance training for competitive swimming. Medicine and Science in Sports and Exercise 25: 952-959, 1993.

33. Tanaka $\mathrm{H}$ and Swensen $\mathrm{T}$. Impact of resistance training on endurance performance. Sports medicine 25: 191-200, 1998.

34. Yanai T. What causes the body to roll in front-crawl swimming? J Appl Biomech 17: 2842, 2001.

35. Yanai T. Stroke frequency in front crawl: its mechanical link to the fluid forces required in non-propulsive directions. Journal of Biomechanics 36: 53-62, 2003.

36. Yanai T. Buoyancy is the primary source of generating bodyroll in front-crawl swimming. Journal of Biomechanics 37: 605-612, 2004.

37. Young WB. Transfer of strength and power training to sports performance. International journal of sports physiology and performance 1: 74-83, 2006. 
418 Figure 2. Time series with ensemble averages for hip roll velocity, shoulder roll velocity, and 419 torso twist velocity at sprint pace and $400 \mathrm{~m}$ pace. Dashed lines represent $95 \%$ confidence 420 intervals calculated using the $t$-value and standard error of the sample mean. Positive values 421 indicate rotation to the swimmer's left (i.e. in the anticlockwise direction when viewing the 422 swimmer from behind) and negative values indicate rotation to the swimmer's right (i.e. in the 423 clockwise direction when viewing the swimmer from behind). Swimmers began these SCs with 424 the right hand. Time series for SCs beginning with the left hand were similar to this figure. 



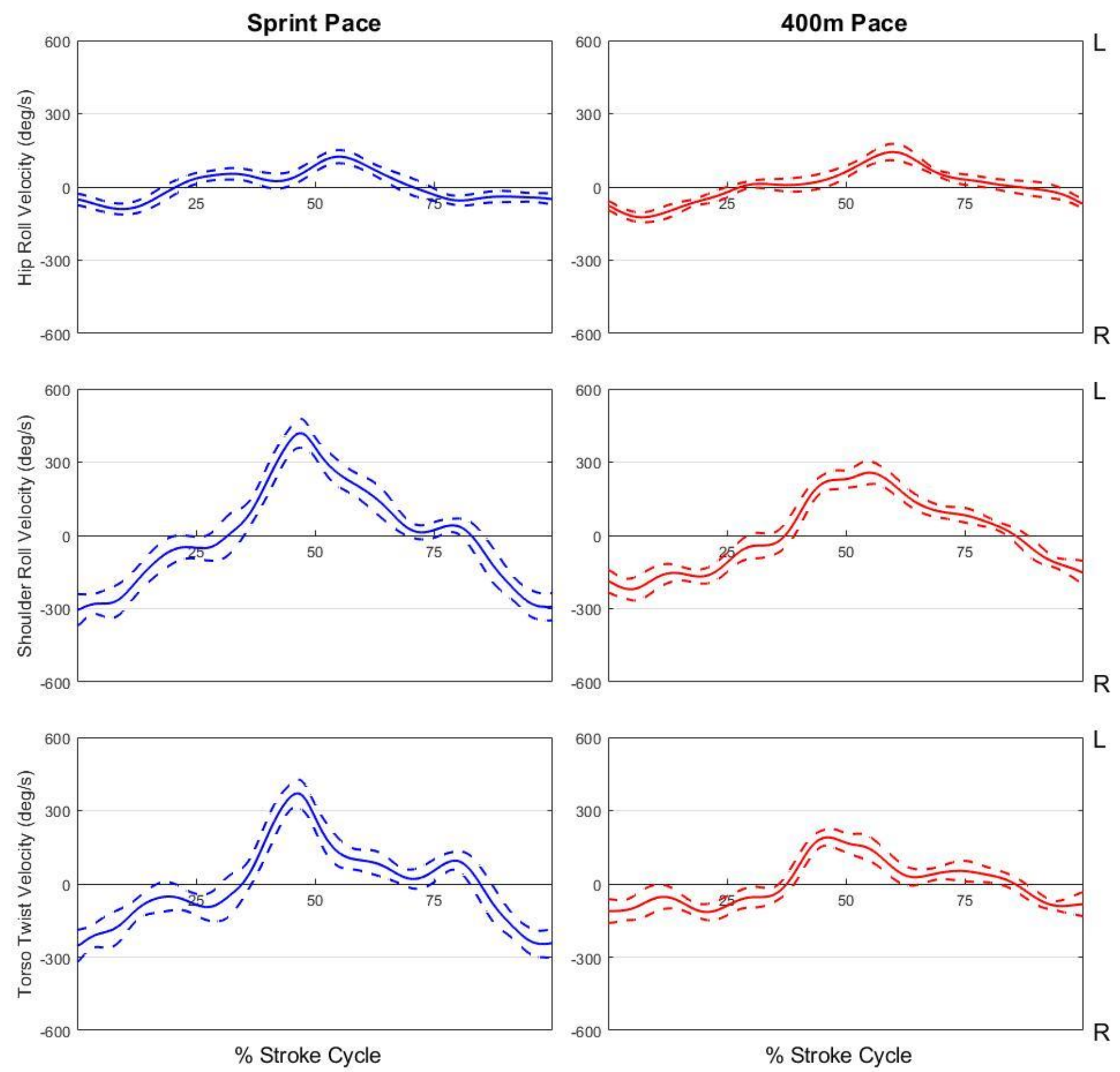
1 Table 1. Stroke frequency, range of hip roll, range of shoulder roll, range of torso twist, average

2 absolute hip roll velocity, average absolute shoulder roll velocity, and average absolute torso

3 twist velocity at sprint pace and $400 \mathrm{~m}$ pace.

\begin{tabular}{|c|c|c|c|c|c|c|}
\hline & \multicolumn{2}{|c|}{ Sprint Pace } & \multicolumn{2}{|c|}{ 400m Pace } & \multirow{2}{*}{$\begin{array}{l}\text { Effect Size } \\
\text { (Cohen's } d \text { ) }\end{array}$} & \multirow{2}{*}{$\begin{array}{c}\text { Power } \\
(n=13)\end{array}$} \\
\hline & Mean & $95 \% \mathrm{CI}$ & Mean & $95 \% \mathrm{CI}$ & & \\
\hline Stroke Frequency (stroke/s) & $0.95 * *$ & 0.04 & 0.70 & 0.04 & 3.73 & 1.0 \\
\hline $\begin{array}{c}\text { Range of } \\
\text { Hip Roll }\left(^{\circ}\right)\end{array}$ & $36.8 * *$ & 3.1 & 49.9 & 5.6 & -1.58 & 1.0 \\
\hline $\begin{array}{c}\text { Range of } \\
\text { Shoulder Roll }\left({ }^{\circ}\right)\end{array}$ & 97.7 & 3.1 & 101.6 & 5.9 & -0.46 & 0.40 \\
\hline $\begin{array}{c}\text { Range of } \\
\text { Torso Twist }\left(^{\circ}\right)\end{array}$ & $78.1 * *$ & 3.4 & 61.3 & 4.7 & 2.23 & 1.0 \\
\hline $\begin{array}{c}\text { Average Absolute } \\
\text { Hip Roll Velocity }(\% / s)\end{array}$ & $75.5^{*}$ & 7.1 & 69.1 & 7.9 & 0.52 & 0.66 \\
\hline $\begin{array}{c}\text { Average Absolute } \\
\text { Shoulder Roll Velocity }(\% / s)\end{array}$ & $190.7 * *$ & 9.9 & 139.2 & 11.5 & 2.92 & 1.0 \\
\hline $\begin{array}{c}\text { Average Absolute } \\
\text { Torso Twist Velocity ( } \% \text { s) }\end{array}$ & $166.3 * *$ & 10.0 & 96.9 & 8.2 & 4.13 & 1.0 \\
\hline
\end{tabular}

